\title{
Fitzpatrick Skin Type V
}

National Cancer Institute

\section{Source}

National Cancer Institute. Fitzpatrick Skin Type V. NCI Thesaurus. Code C74573.

Skin which is brown and it burns very rarely when exposed to the sun. It is usually seen in people of Middle Eastern origin. 